\title{
Comparative Sexuality in Agaricus Species
}

\author{
By T. J. ELLIOTT \\ Glasshouse Crops Research Institute, Littlehampton, Sussex BN16 3PU
}

(Received 20 February 1978)

The sexuality of the wild four-spored Agaricus species, A. bitorquis, A. nivescens, A. macrosporus and $A$. silvicola, was investigated and compared with that of the two-spored commercially cultivated mushroom, $A$. bisporus. For each of the wild species, 20 single spore isolates were mated in all combinations. Heterokaryosis was considered to have occurred when novel mycelial growth was apparent at the junction zones. Unifactorial heterothallism was confirmed in A. bitorquis and shown to occur in A. nivescens and A. macrosporus. There was no indication that nuclear migration occurred. Additional alleles of the mating-type factor were detected in other stocks of these three species. In A. macrosporus, heterokaryosis was enhanced by the addition of activated charcoal to the culture medium.

Heterokaryosis was confirmed where possible by fruiting tests in conditions used for growing A. bisporus. Agaricus bitorquis and A. macrosporus fruited but A. nivescens did not. All matings of $A$. silvicola isolates were negative which, together with fruiting data, suggests that this species may be homothallic. There was no indication of heterokaryosis in interspecific matings amongst these species or with $A$. bisporus.

The expression of sexuality in $A$. bitorquis, $A$. nivescens and $A$. macrosporus is contrasted with that in $A$. bisporus. It is suggested that $A$. bisporus evolved from a species of the macrosporus/nivescens type and that the constitutive heterokaryosis of $A$. bisporus spores may be leading to true homothallism.

\section{INTRODUCTION}

Incompatibility mechanisms are widespread in the higher fungi. The Homobasidiomycetidae, in particular, are characteristically heterothallic with compatibility controlled by one, two or even three factors (Raper, 1966; Jurand \& Kemp, 1973). The genus Agaricus, which belongs to this group, has been little investigated in relation to incompatibility systems. This is surprising in view of the commercial importance of Agaricus bisporus, the cultivated mushroom.

Heterothallism has been reported in A. bisporus itself (Miller, 1971; Raper, Raper \& Miller, 1972; Elliott, 1972) and in A. bitorquis (Raper, 1976). In both species compatibility seems to be controlled by a single factor. Homothallism has been reported in A. campestris, the common field mushroom (Klushnikova, 1939).

This paper reports an investigation of $A$. bitorquis and of three other wild species, $A$. silvicola, $A$. nivescens and $A$. macrosporus, whose incompatibility systems have not previously been studied. The work was undertaken to compare the breeding behaviour of these species with that expressed in $A$. bisporus. 


\section{METHODS}

Taxonomy. The identification of species in the genus Agaricus can be difficult and specific names given to collections from the wild should generally be accepted with caution. It has been shown, for example, that collections identified as three distinct species are all interfertile with $A$. bitorquis (Raper, 1976). Of the four species studied here, three are distinct and readily recognized, i.e. A. bitorquis, A. silvicola and A. macrosporus. The descriptions and plates of Møller (1949-1953) and Essette (1964) for $A$. nivescens agree closely with the fourth species discussed here. Even so, the identification of this species as $A$. nivescens should be regarded as tentative as there are several similar species.

Agaricus bitorquis, A. silvicola, A. nivescens and A. macrosporus are four-spored in contrast to the twospored $A$. bisporus. Clamp connections have not been seen in any of these species.

Nomenclature and strains. Mycelia of the wild species were initially established by culturing sporophore tissue; these were given a w coding, e.g. w2, w7. Single spore cultures of the same species obtained by serial dilution of spore suspensions are coded by a number suffix following the w coding, e.g. w2-5, w7-15. Other single spore cultures derived from individual spores isolated directly from gill surfaces have an A coding. Mating-types are indicated in parentheses following the strain designation. The following stocks were used.

Agaricus bitorquis (Quél.) Sacc. (w2): stock I, collected by Professor P. Heinemann at Wezembeck, Belgium, and identified and supplied by him; stock II, collected by J. Pegler at Storrington, Sussex, and identified by the author.

Agaricus silvicola (Vitt.) Peck (w3): collected in the grounds of the Glasshouse Crops Research Institute (G.C.R.I.) and identified by the author.

Agaricus nivescens (F. H. Møller) F. H. Møller (w6): stock I, collected at Rustington, Sussex, and identified by the author; stocks II and III, collected at Wisborough Green, Sussex, by R. H. Gaze, and identified by the author.

Agaricus macrosporus (F. H. Møller \& J. Schaeff.) Pilát (w7): stocks I and II, collected in the grounds of G.C.R.I. from different sites and identified by the author.

Agaricus bisporus (J. Lange) Imbach: the homokaryotic strains of $A$. bisporus were from the G.C.R.I. culture collection.

Media. Complete yeast extract medium (CYM) as described by Raper et al. (1972) and charcoal-CYM were used. Activated charcoal was incorporated at $0.05 \mathrm{~g} \mathrm{l}^{-1}$. The $\mathrm{pH}$ was adjusted to 7.5 by the addition of sodium hydroxide before autoclaving. All media were autoclaved for 20 min at 1 bar.

Germination. Spores of all species were germinated at $25^{\circ} \mathrm{C}$ in the presence of actively growing mycelium of $A$. bisporus. It was hoped that volatile substances produced by the mycelium would stimulate germination (Lösel, 1964). Single spores were isolated by micromanipulation and incubated individually on sterile cellophane strips around a single central inoculum (Elliott, 1972). Spore dilutions were incubated as 'doubleplates'. The bottom half of one Petri dish containing the spores was attached to the inverted bottom half of a second dish in which there was an actively growing 2 to $3 \mathrm{~cm}$ colony of $A$. bisporus. The dishes were joined using adhesive tape, a gap of approximately $2 \mathrm{~cm}$ being left to permit air exchange.

Mating tests. Tests for compatibility within each species were carried out by growing pairs of isolates side by side and observing the zones of hyphal intermingling. Heterokaryosis was considered to have taken place when new and distinct growth was apparent in these zones. Such matings were scored as positive. Pairings in which there was no new growth in the junction zone were scored as negative. Plates were kept for at least 2 months for regular checking.

When more than one stock of a species was available both intra- and interstock matings were made. Interspecific matings were also attempted. Ten homokaryons of the species under test were mated with tester homokaryons of each of the other species. These tester homokaryons were of opposite mating-type and were chosen for their uniform growth and consistent mating behaviour. Matings were made on both CYM and charcoal-CYM, the homokaryons being arranged so that both intra- and interspecific confrontations could be assessed (Fig. 1).

Fruiting. Heterokaryosis was confirmed where possible by fruiting tests. They were carried out in the standard conditions used for growing $A$. bisporus. Attempts were also made to fruit single spore isolates.

\section{RESULTS}

\section{Agaricus bitorquis (w2)}

Intrastock mating. Twenty-five single spore isolates, A24, A25 and A27 to A49, were established from stock I, and 10 of these were mated in all combinations. Heterokaryotic mycelium was apparent in some matings. The isolates were thus divided into two groups, 


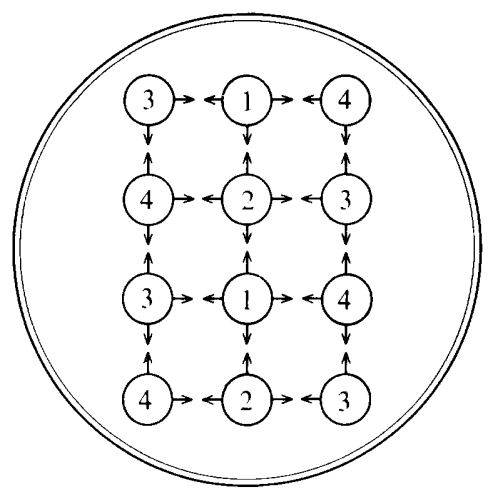

Fig. 1. Arrangement of homokaryons of Agaricus spp. in interspecific mating tests: 1 and 2 are tester homokaryons; 3 and 4 are single spore isolates of the species under test. Arrows indicate hyphal confrontation zones.

with six isolates of one mating-type and four of the other. Fifteen of the 24 expected positive matings were positive. Heterokaryotic mycelia from 12 of the positive matings were subcultured for fruiting tests.

A further 77 isolates obtained from a spore suspension of stock I were mated with isolates A30 and A38 which had been ascribed mating-types (A2) and (A1), respectively: 40 were positive only with the (A1) tester, 12 were positive only with the (A2) tester, one (w2-22) was positive with both testers and 24 mated with neither tester.

To eliminate any doubt as to the control of compatibility in this species, 20 isolates obtained from stock I by serial dilution were mated in all combinations on CYM. Duplicate plates of each mating were made and two pairs of isolates were inoculated on each plate with approximately $10 \mathrm{~mm}$ between isolates. This gave four contrasting hyphal confrontations per plate and a total of eight possible positives per mating. The formation of a heterokaryon was assumed if vigorous, fluffy mycelial growth was observed in the zone of hyphal intermingling. This occurred 1 or $2 \mathrm{~d}$ after hyphal contact in some cases but it was delayed for several weeks in others. The production of heterokaryotic mycelium varied greatly and positive matings could be quantitatively distinguished into three categories: (i) growth of heterokaryotic hyphae restricted to zone of hyphal intermingling (22\%); (ii) vigorous growth of heterokaryon with some overgrowth of parental homokaryons $(36 \%)$; (iii) vigorous growth of heterokaryon associated with the production of fruit-body initials, parental homokaryons being completely overgrown $(42 \%)$. In most positive matings all confrontations produced heterokaryotic mycelium, but not always synchronously. In a few cases $(<5 \%)$, however, only one or two of the confrontation zones produced obviously heterokaryotic mycelium.

If all of these positive matings are taken to indicate compatibility, the pattern of matings is entirely consistent with control by a single factor. The mating-types of the 20 isolates tested were: type I, isolates w2-2, -3, -4, -5, -7, -11, -14, -21, -26, -28 and -29 ; type II, isolates $\mathrm{w} 2-8,-12,-16,-17,-18,-19,-20,-23$ and -30 . Five of the expected positive matings were at first scored as negative. In each case, the apparent absence of heterokaryosis was related to isolates $\mathrm{w} 2-14$ and w2-30 which both produce distinctive dense and compact mycelium. These two isolates were generally poor maters in that little heterokaryotic mycelium was formed in matings involving them. When the five obscure matings involving these two isolates were repeated, two of them were scored as positive and so only matings w2-14 $\times w 2-20$, w2- $26 \times w 2-30$ and w2-28 $\times w 2-30$ failed to give the expected positive results. None of the isolates showed ambivalent positive mating behaviour.

Ten homokaryons of stock II were intermated to determine the number of mating-types present; again two were detected. 
Interstock mating. Four homokaryons of stock II, two of each mating-type, were mated with tester homokaryons of stock I. All matings were positive indicating that there are four different mating-type alleles in these two stocks of $A$. bitorquis.

Fruiting. Heterokaryons of this species have a distinctive morphology and produce fruitbody initials in axenic culture. A few had some spore-bearing tissue after prolonged incubation. Both stocks I and II and heterokaryons derived from them fruited in the conditions used for $A$. bisporus although the optimum temperature for $A$. bitorquis is higher (Raper, 1976). Homokaryotic mycelia did not fruit. Of the 12 heterokaryons produced in the initial mating study, seven fruited within 30 to $50 \mathrm{~d}$ after casing, but the remaining five did not fruit.

\section{Agaricus silvicola (w3)}

Mating data. Ten single spore isolates obtained by serial dilution were mated in all combinations. There were no interactions between any isolates and all matings were recorded as negative. A further 20 single spore isolates were mated in all combinations on CYM. Of the 190 matings, all were negative. In 182 of them the mycelia of adjacent isolates became confluent. In the other eight, the junction zone was still apparent after prolonged incubation but there was no sign of the production of novel mycelial growth in this zone.

Fruiting. Attempts were made to fruit the 10 original single spore isolates. Isolates w3 $-1,-6,-7$ and -14 produced fruit-body initials on the grain used during the preparation of spawn. Isolates w3-1, -6 and -7 produced abnormal sporophores 35 to $40 \mathrm{~d}$ after casing. Stipe elongation seemed normal but pileus expansion and development were restricted.

\section{Agaricus nivescens (w6)}

Intrastock mating. Twelve tetrads were isolated by micromanipulation from stock I. One complete tetrad germinated and matings between the four homokaryons clearly showed the growth of heterokaryotic hyphae at the junction zone in four of the six possible matings. The four heterokaryons produced were subcultured for fruiting tests. Ten other isolates from the same sporophore were mated with members of the tetrad and two mating types were found in the proportion $6: 4$.

Additional sporophores were collected from the same site in the following season and 30 more tetrads were isolated. Of the 58 single spore germlings obtained, the first 20 which germinated were mated in all combinations to extend the data on mating interactions for this species. New growth of white fluffy mycelium at the zone of hyphal intermingling was taken to indicate heterokaryosis (Fig. 2). In this species the production of heterokaryotic mycelium again varied greatly in timing and intensity and was often accompanied by the development of dark brown pigmentation in the medium. Some matings were vigorous, whilst in others the heterokaryotic mycelium was restricted to localized tufts in one or two junction zones. Single spore isolates of this species often produced slow-growing, irregularly shaped colonies on CYM. This limited the zones of hyphal intermingling and made matings more difficult to score. Nevertheless, the isolates could be clearly divided into two groups on the basis of their mating behaviour indicating a unifactorial control of compatibility.

The data with the isolates grouped according to mating-type are shown in Table 1. Isolates A131, A145 and A146 were particularly slow growing. Although some matings, especially those involving A131, A145 and A146, could not be scored due to the disparate growth of the homokaryons, no spurious positive results were recorded.

The growth of single spore isolates from stocks II and III was variable but generally poor. The pattern of matings between these isolates was incomplete but the positive matings within both stocks were consistent with unifactorial heterothallism.

Interstock mating. Matings of homokaryons of contrasting mating-type of stock I with homokaryons of stocks II and III were difficult to score. However, three homokaryons of stock III gave positive matings with both tester homokaryons of stock I suggesting the presence of at least one additional mating-type allele. The few positive matings of stock II 
Table 1. Relationships of 20 single spore isolates of Agaricus nivescens (w6) grouped according to mating-type

The single spore isolates are all $\mathrm{A}$ isolates. $\bullet$, Heterokaryosis; $\bigcirc$, no interaction. Some combinations could not be scored.

Type I

Type II

$\overbrace{132133135136}^{140} 144 \quad 145146148131134137138139141 \quad 142143147149150$

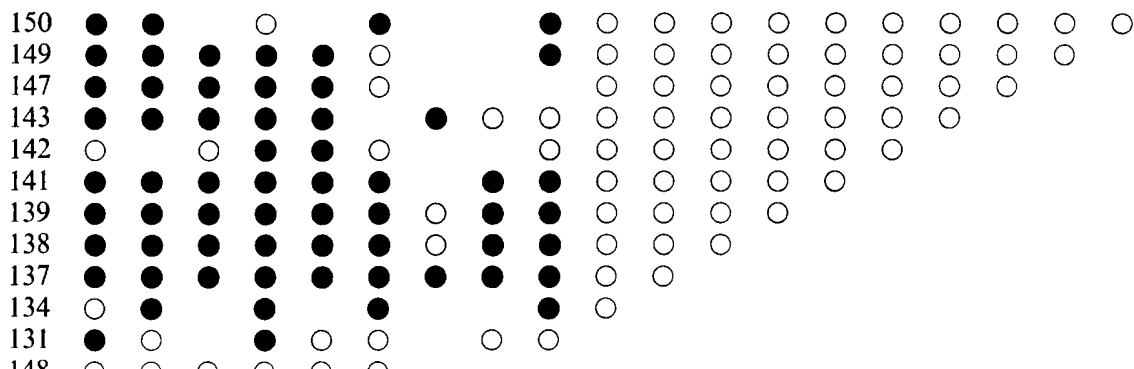

$\begin{array}{llllllll}148 & 0 & 0 & 0 & 0 & 0 & 0\end{array}$

$\begin{array}{llllll}146 & 0 & 0 & 0 & 0 & 0\end{array}$

$\begin{array}{lllll}145 & 0 & 0 & 0 & 0\end{array}$

$\begin{array}{lllll}144 & 0 & 0 & 0 & 0\end{array}$

$\begin{array}{llllll}140 & \circ & \circ & \bigcirc & 0\end{array}$

$\begin{array}{lllll}136 & 0 & 0 & 0\end{array}$

$135 \bigcirc \bigcirc$

133

132

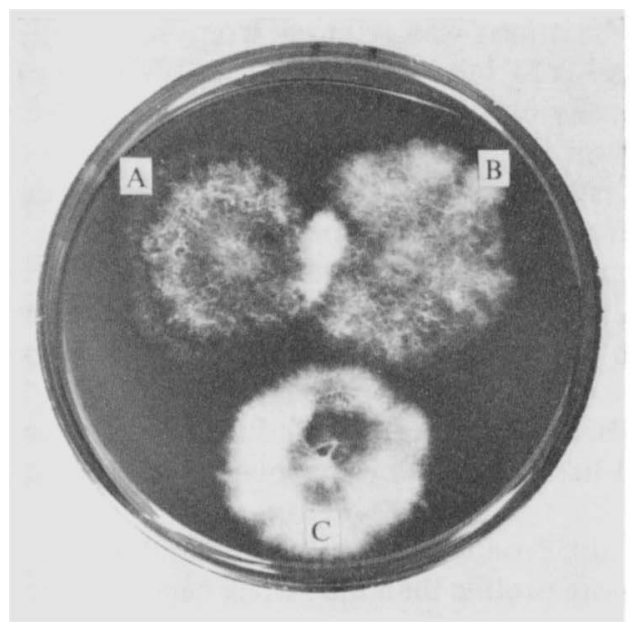

Fig. 2

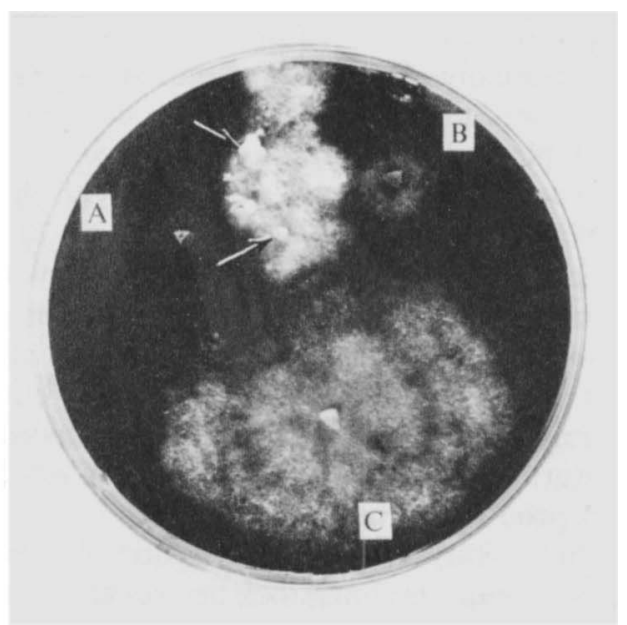

Fig. 3

Fig. 2. Growth of compatible homokaryons (A and B) of Agaricus nivescens and the heterokaryon (C). Heterokaryosis can be seen in the junction zone between the homokaryons.

Fig. 3. Growth of compatible homokaryons (A and B) of Agaricus macrosporus and the heterokaryon (C) formed on charcoal-CYM. Sporophore initials (arrowed) have only formed on the heterokaryotic mycelium between the homokaryons.

homokaryons with the testers were consistent with the same alleles being present in stock II as in stock I.

Fruiting. Fruit-body initials were not seen in axenic culture. In attempts to fruit the four heterokaryons produced by intermating the original tetrad, all four produced primordia 50 to $60 \mathrm{~d}$ after casing but no mature sporophores. Attempts to fruit homokaryons were 
limited by their poor ability to colonize grain spawn and mushroom compost. Heterokaryons were usually good compost colonizers.

\section{Agaricus macrosporus (w7)}

Intrastock mating. Thirteen single spore isolates, A92 to A104, were established from stock I. Seven of these with similar growth rates were mated in all combinations on CYM. Certain matings produced conspicuous heterokaryotic mycelium but there was no consistent pattern amongst the isolates and they could not be readily divided into discrete groups. Seventy-two single spore isolates derived from the serial dilution of a spore suspension of stock II were mated on CYM with compatible isolates A94(A2) and A95(A1) of the original stock I sample. Only 16 positive matings were recorded. Three isolates were positive with both testers (see interstock mating below).

The incorporation of activated charcoal into the medium had been shown to enhance heterokaryosis in Rhizoctonia (Butler \& Bolkan, 1973) so the matings of the original sample were repeated on charcoal-CYM. The results clearly showed that two mating-type alleles were present. The formation of heterokaryotic mycelium occurred earlier and was much more extensive in the presence of charcoal (Fig. 3) and the first positive matings were scored after $10 \mathrm{~d}$.

A larger sample was then tested to check the effect of charcoal and to confirm that mating competence was controlled by a single factor. Twenty isolates chosen at random from stock II were mated in all combinations on both CYM and charcoal-CYM. On the basis of positive matings the isolates could be clearly divided into two groups. On CYM the number of expected positive results scored as negative was high, but on charcoal-CYM a much more complete pattern of matings was revealed (Table 2). Assuming control by a single factor, the failure rate (Ginns, 1974), i.e. the number of expected positive matings scored as negative expressed as a percentage of the total positive matings, was reduced from 42 to $10 \%$. Occasionally a mating was scored as positive on CYM but not on charcoal-CYM. As in $A$. bitorquis and $A$. nivescens, the timing and intensity of heterokaryon formation were very variable and the mating-type ratio of $16: 4$ is a poor fit to the expected $1: 1$ ratio.

Another 39 isolates were mated with the two tester strains w7-6 and w7-15 on charcoalCYM. With these isolates there was a 20:19 segregation of the mating-type factors.

Interstock mating. The 20 homokaryons from stock II which had been mated in all combinations were mated against isolates A94(A2) and A98(A1) of stock I. All matings were positive with both testers showing that although stocks I and II originated from adjacent sites at the G.C.R.I. they possess different alleles of the mating-type factor.

Fruiting. Some heterokaryons produced fruit-body initials in the mating zone in axenic culture (Fig. 3). However when such established heterokaryons were subcultured they did not produce initials.

Heterokaryons fruited whilst homokaryons did not. Productivity was.low in standard mushroom composts; interstock heterokaryons were more prolific than intrastock heterokaryons.

The characteristics of the incompatibility systems of $A$. bitorquis, $A$. nivescens and A. macrosporus are summarized in Table 3.

\section{Interspecific matings}

Agaricus bitorquis, A. nivescens and A. macrosporus were reciprocally intermated. Matings were also attempted between the wild species and $A$. bisporus. All the intraspecific matings between testers of $A$. bitorquis, $A$. nivescens and $A$. macrosporus were positive. Interspecific matings are considered below.

bitorquis $\times$ nivescens. No matings were scored as positive. Two matings were scored as indeterminate. The growth of adjacent isolates tended to overlap.

bitorquis $\times$ macrosporus. No matings were scored as positive. On charcoal-CYM there was a demarcation zone in which growth was inhibited between isolates. 
Table 2. Relationships of 20 single spore isolates of Agaricus macrosporus (w7) grouped according to mating-type on CYM and charcoal-CYM

The single spore isolates are all w7-isolates. Heterokaryosis on both media; $\boldsymbol{\nabla}$, heterokaryosis on CYM only; $\boldsymbol{\Lambda}$, heterokaryosis on charcoal-CYM only; $\bigcirc$, no interaction on both media.

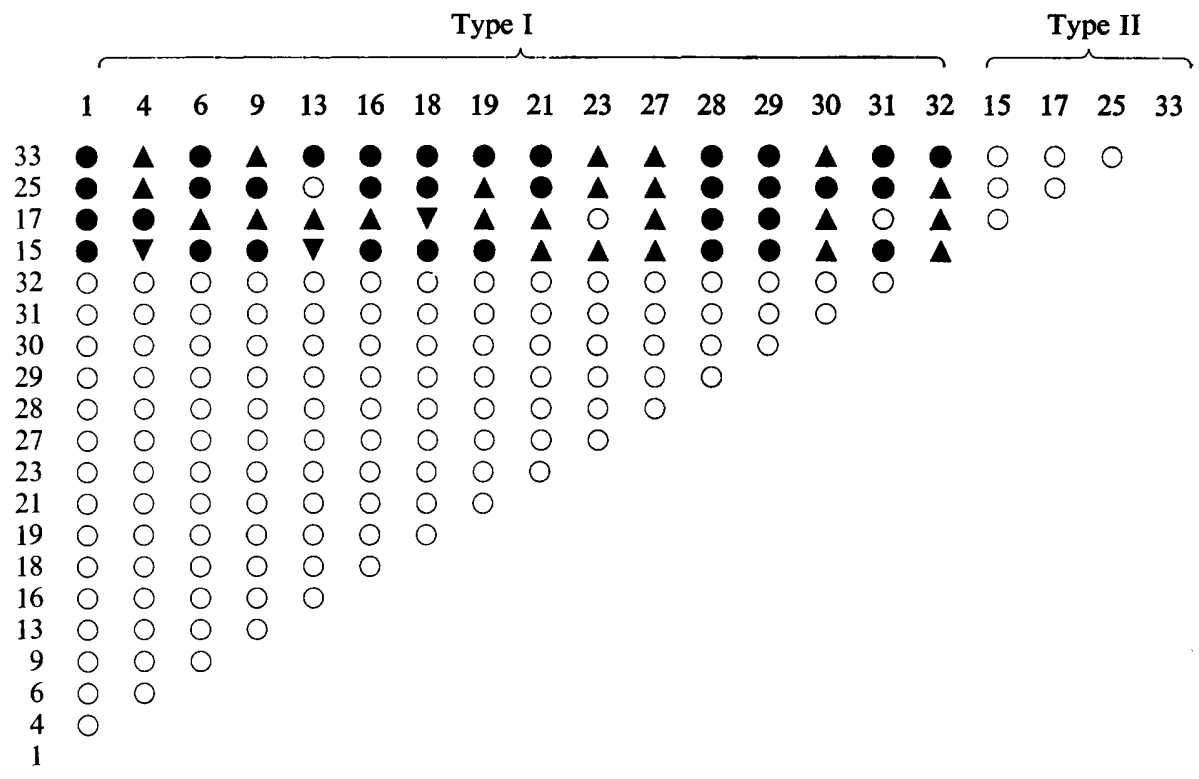

Table 3. Sexuality in Agaricus species and, for comparison, in Polyporus palustris*

Sexuality

Polyporus palustris

Unifactorial heterothallic

\section{A. bitorquis}

Unifactorial heterothallic
A. macrosporus

A. nivescens

Unifactorial heterothallic
A. bisporus

'Secondarily homothallic' (Unifactorial heterothallic)
Monokaryons

Dikaryons

Distinct mating interactions Nuclear migration Spores/basidium

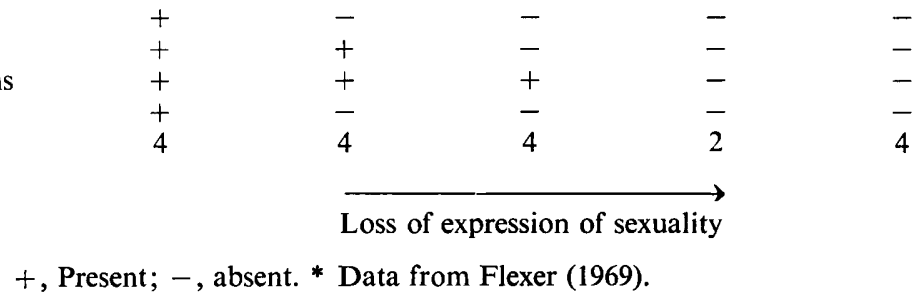

nivescens $\times$ macrosporus. No matings were scored as positive. Some matings were difficult to assess due to contrasting mycelial growth.

bisporus $\times$ bitorquis. No matings were scored as positive. On charcoal-CYM there was a demarcation zone between the colonies.

bisporus $\times$ silvicola. No matings were scored as positive. In some matings, particularly on charcoal-CYM, there was a zone of restricted growth between isolates.

bisporus $\times$ nivescens. No matings were scored as positive. The $A$. nivescens homokaryons grew poorly on charcoal-CYM.

bisporus $\times$ macrosporus. Two matings were scored as weakly positive on CYM but subcultures from the mating zone were of the A. bisporus type. There was a zone of inhibited growth between the isolates, especially on charcoal-CYM. 


\section{DISCUSSION}

Studies on Agaricus species other than A. bisporus are of particular interest for several reasons. Such species may have potential for cultivation in their own right. They might be used as sources of genetic variation that could be introduced into $A$. bisporus and could give a better understanding of the biology of the commercial mushroom. Agaricus bitorquis is already grown commercially and in hot climates offers an acceptable alternative to $A$. bisporus. The potential of $A$. macrosporus has already been explored by Uzonyi (1965) and she reported yields of $29 \mathrm{~kg} \mathrm{~m}^{-2}$ which compare favourably with those of $A$. bisporus. The potential of $A$. silvicola and $A$. nivescens remains to be determined. Both species are difficult to fruit but the difficulties are probably due to cultural methods rather than genotypic limitations. Fruiting of $A$. arvensis, which the author believes to be synonymous with $A$. silvicola, has been reported in culture (Couvy, 1974).

The species described here are distinct on the basis of fruit-body characteristics, colony morphology and mycelial interactions. Interspecific matings between homokaryons may be a guide to species relationships. A microscopic examination for the presence of hyphal fusions and cytoplasmic lethality could help in determining the relationships of the species (Kemp, 1975). It is possible that auxotrophs could be used to force the production of 'transient heterokaryons' as described between $A$. bitorquis and A. bisporus (Raper, 1976). However, the above data show that none of the four-spored species are interfertile with each other or with $A$. bisporus.

The data obtained for $A$. bitorquis are in full agreement with those of Raper (1976). Agaricus bitorquis shows a multiallelic unifactorial heterothallism and compatible homokaryons visibly interact at their junction zones to produce morphologically distinct heterokaryons. Nuclear migration was not detected even by using auxotrophic markers. Raper (1976) also showed that although homokaryons are multinucleate there is a tendency towards conjugate nuclear divisions and thus dikaryosis in the heterokaryon. This has since been confirmed in a comparative cytological study within the genus Agaricus (Elliott \& Hou, unpublished results).

Raper (1976) also found some evidence of intrafactor recombination in A. bitorquis. The ambiguous nature of isolate w2-22 which produced heterokaryotic mycelium with sibling isolates of contrasting mating-type may support this contention. A more detailed analysis would be necessary to establish beyond any doubt that it occurs. The fruiting data indicate that other factors are segregating which influence sporophore production.

Agaricus macrosporus and A. nivescens can be considered together. All the mating and fruiting data are consistent with multiallelic unifactorial heterothallism. In both species heterokaryosis, as judged by the formation of distinctive mycelium and by fruiting trials, is limited to the junction zone between compatible isolates. The heterokaryon of $A$. macrosporus has a distinct and recognizable colony morphology when subcultured, but the morphology of the A. nivescens heterokaryon is nearer to that of its homokaryons (Figs 2 and 3 ). Transfers made from the mycelium either side of the junction line in a mating test have the morphology of the homokaryons. There would appear to be no nuclear migration but more extensive tests are necessary to prove this. In $A$. macrosporus the incorporation of charcoal into the medium was useful in the detection of heterokaryons. The mode of action of the charcoal is not understood but its effect is probably due to its adsorbent properties. No within-stock homokaryons of $A$. macrosporus or $A$. nivescens were doubly positive with sibling homokaryons of contrasting mating-type. The mating behaviour of large numbers of sibling isolates would need to be established to determine if intrafactor recombination occurs in these species.

The limited data for $A$. silvicola suggest homothallism but the partial fruiting response of some single-spore isolates may represent the homokaryotic fruiting of a heterothallic species. Agaricus silvicola may be heterothallic but with limited heterokaryosis as in $A$. bisporus. 
Single spore isolates vary in growth rate and colony morphology which might be evidence of heterothallism. However, restricted heterokaryosis seems unlikely in a normally four-spored species which does not produce asexual spores. Vigour would be expected to be associated with heterokaryosis because fruiting and basidiospore production depend on the competitive growth of heterokaryons over homokaryons.

The two-spored A. bisporus is heterothallic but differs from $A$. bitorquis, A. nivescens and A. macrosporus. Its spores are normally heterokaryotic and fertile. However, homokaryons have been obtained from aberrant three- and four-spored basidia and have been used to demonstrate the basic heterothallism of this species (Miller, 1971 ; Elliott, 1972). The mating data available are consistent with a unifactorial control of mating competence (Raper et al., 1972; Miller \& Kananen, 1972) although a reinterpretation of the data has been presented supporting a bifactorial control (Ginns, 1974). Homokaryons and heterokaryons are not morphologically distinct and both are multinucleate (Evans, 1959; Elliott, 1971). Heterokaryon formation is limited to the zone of hyphal intermingling between compatible homokaryons but there is no visible indication of its occurrence. Agaricus bisporus is essentially an inbreeding species although some heterozygosity may be conserved by the non-random orientation of the meiotic spindles (Evans, 1959). However, the amount of outbreeding which can occur is not known. Two-sporedness is generally seen as a short-term device to limit outbreeding (Koltin, Stamberg \& Lemke, 1972). Its evolutionary significance has been considered by Kemp (1974) who suggested that it could be a factor in speciation and that two-spored species, although probably derived from four-spored species, might evolve to give new four-spored species.

The species of Agaricus which have been shown to be heterothallic are A. bisporus, $A$. bitorguis, $A$. nivescens and $A$. macrosporus and all are unifactorial. The mode of action of this single factor varies between these species. In $A$. bitorquis, it controls heterokaryon formation and regulates nuclear number within the heterokaryon to produce a dikaryon. In $A$. macrosporus and A. nivescens, it controls heterokaryon formation but heterokaryons of both species are multinucleate (Elliott \& Hou, unpublished results) and dikaryotic cells are rarely found. In A. bisporus, the function of the mating-type factor is even further reduced. Heterokaryosis is not apparent and the cells of both homokaryons and heterokaryons are multinucleate. Agaricus bisporus is possibly derived from a four-spored species of the macrosporus/ nivescens type from which some of the specificity of the mating-type factor has been lost. A loss of expression of genes regulated by the mating-type factor would not necessarily impair the functioning of a species which produces dikaryotic spores.

An evolutionary progression can be postulated from an ancestral bitorquis type to a macrosporus/nivescens type to the two-spored bisporus with a decreasing expression of sexuality. Expression of sexuality in $A$. bisporus can be considered residual. The next step in the evolutionary sequence for $A$. bisporus could be four-spored true homothallism as shown by $A$. campestris and possibly $A$. silvicola. Although homothallism may reduce the longterm survival prospects of a species it could favour short-term abundance in a stable environment. The proposed progression is shown in Table 3 where the Agaricus species are contrasted with a more typical unifactorial heterothallic basidiomycete, Polyporus palustris (Flexer, 1969).

I would like to thank Mrs J. B. Batts for technical assistance and Mr L. A. Darby and Dr F. A. Langton for their help in preparing this paper. 


\section{REFERENCES}

Butler, E. E. \& Bolkan, H. (1973). A medium for heterokaryon formation in Rhizoctonia solani. Phytopathology 63, 542-543.

Couvy, J. (1974). La fructification d'Agaricus arvensis Fries Schaeffer en culture pure. Action de la temperature et de la lumière. Bulletin de la Société mycologique de France 90, 81-88.

Elliott, T. J. (1971). Plant Breeding Research Summary. Annual Report of the Glasshouse Crops Research Institute 1970, 62-64.

ElliotT, T. J. (1972). Sex and the single spore. Mushroom Science 8, 11-18.

EssetTe, H. (1964). Atlas Mycologiques I. Les Psalliotes. Paris: Editions Paul Lechevalier.

Evans, H. J. (1959). Nuclear behaviour in the cultivated mushroom. Chromosoma 10, 115-135.

FleXer, A. S. (1969). Bipolar incompatibility in the hymenomycete, Polyporus palustris. American Journal of Botany 56, 410-417.

GinNs, J. H. (1974). Secondarily homothallic Hymenomycetes: several examples of bipolarity are reinterpreted as being tetrapolar. Canadian Journal of Botany 52, 2097-2110.

JuRAND, M. K. \& KeMP, R. F. O. (1973). An incompatibility system determined by three factors in a species of Psathyrella (Basidiomycetes). Genetical Research, Cambridge 22, 125-134.

KEMP, R. F. O. (1974). Bifactorial incompatibility in the two-spored basidiomycetes Coprinus sassii and C. bilanatus. Transactions of the British Mycological Society 62, 547-555.

KEMP, R. F. O. (1975). Breeding biology of Coprinus species in the section Lanatuli. Transactions of the British Mycological Society 65, 375-388.
Klushnikova, E.S. (1939). The wild Psalliota campestris, its sexual character and its relation to the cultivated mushroom. Bulletin de la Société des naturalistes de Moscou, N.S. 48, 53-58.

Koltin, Y., StamberG, J. \& Lemke, P. A. (1972). Genetic structure and evolution of the incompatibility factors in higher fungi. Bacteriological Reviews 36, 156-171.

LösEL, D. M. (1964). The stimulation of spore germination in Agaricus bisporus by living mycelium. Annals of Botany 28, 541-554.

Miller, R. E. (1971). Evidence of sexuality in the cultivated mushroom, Agaricus bisporus. Mycologia 63, 630-634.

Miller, R. E. \& Kananen, D. L. (1972). Bipolar sexuality in the mushroom. Mushroom Science $\mathbf{8}$, 713-718.

Møller, F. H. (1949-1953). Danish Psalliota species. Preliminary studies for a monograph on the Danish Psalliotae, I \& II. Freisia 4, 1-60, 135-220.

RAPER, C. A. (1976). Sexuality and life-cycle of the edible, wild Agaricus bitorquis. Journal of General Microbiology 95, 54-66.

RAPER, C. A., RAPER, J. R. \& Miller, R. E. (1972). Genetic analysis of the life-cycle of Agaricus bisporus. Mycologia 64, 1088-1117.

RAPER, J. R. (1966). Genetics of Sexuality in Higher Fungi. New York: The Ronald Press.

UzonyI, A. L. (1965). Investigations into some conditions for spore germination, and data on the establishment of growing conditions for Agaricus macrosporus. Mushroom Science 6, 65-75. 